\title{
REVIEW OF DESIGN STRATEGIES OF DUAL/TRI-BAND ANTENNAS FOR GPS AND IRNSS APPLICATIONS
}

\author{
Naik. $\mathbf{P}^{1}$, Ruparelia. $\mathrm{S}^{2}$, Bhatt. $\mathrm{N}^{3}$ and Mahajan. $\mathrm{M}^{\mathbf{4}}$ \\ ${ }^{1,2,3}$ Department of Electronics and Communication Department, C.K. Pithawala College of Engineering and Technology, India \\ ${ }^{4}$ Satellite Communications Antenna Division, ISRO, Gujarat, India
}

\begin{abstract}
In era of ever increasing demand of wireless communications, domain of antenna design has progressed rapidly and gained a lot of attention among researchers and academicians. Over past few decades, it has catered the needs of society and technocrats by proving itself as one of the main driving forces behind recent advancements in wireless communication. Especially in the GNSS applications, for civilian and military use, antenna design has played a vital role. Thus, a humble attempt is made in this article to provide a glance of globally used antenna for the GNSS applications. A spiral antenna. Various aspects of antenna characteristics have been described that gives insight of developments carried out in the field of spiral antenna design and important conclusions are drawn from the comparisons made for their future use. Comparative reviews between various aspects of antenna designs reveal the fact about promising performance of spiral based antenna in developing dual/tri band applications for GPS as well as IRNSS.
\end{abstract}

\section{Keywords \\ IRNSS, Tri-Band, Circular Polarization, Spiral}

\section{INTRODUCTION}

Global Navigation Satellite System (GNSS) is catering global coverage with positioning and timing data to find out the location of the user. GNSS includes Global Positioning System (GPS) from USA, GLObal NAvigation Satellite System (GLONASS) from Russia, Beidou Navigation Satellite System from China. Among all, Global Positioning System (GPS) is a space-based satellite navigation system which was designed, developed and controlled by U.S government that yields location, velocity and time information of users across the globe [1]. Combination of the L1 band $(1575.42 \mathrm{MHz})$, L2 band $(1227.60 \mathrm{MHz})$ and L5 band $(1176.45 \mathrm{MHz})$ in GPS derives improved availability and accuracy for highly robust services [1].

Certain limitations are evident with usage of GPS in Indian and also in Asia-Pacific regions. For some complex and hostile situations, GPS alone cannot meet the accuracy that in turn leads to poor navigation and tracking. In order to provide better accuracy, a new surveillance system proposed called Indian Regional Navigation Satellite System (IRNSS). IRNSS is an autonomous regional navigation system developed by Indian Space Research Organization (ISRO). The entire constellation of IRNSS needs seven satellites. This system exhibits accuracies of up to $10 \mathrm{~m}$ for Indian land region and $20 \mathrm{~m}$ for Indian Ocean region.
The coverage area can be extended up to $1500 \mathrm{~km}$ from the primary Indian region. IRNSS has high accuracy in position, time and velocity information in real time applications. IRNSS has the frequency bands of L5 (1176.45MHz) and S (2492.08MHz) [2]. These bands and GPS (L1) making it tri-band operation required for Indian context wherein both of these services are required to be provided. In literature tri-band in GNSS context, it is taken as L1, L2 and L5 band. However in the context of Indian scenario, it is L1, L5 and S band.

Antennas have brought a revolution in the field of wireless communication systems. Since the earlier antenna systems not focused much on the designing of the antenna in the overall system analysis but now a days it is considered to be one of the most important factors while selecting any antenna system, as the success and failure of the system relies heavily on the design of the antenna [3]. The availability of limited space and weight onboard of IRNSS receiver is a major challenge in antenna designing.

Selection of appropriate antenna for GNSS receiver highly depends on the polarization of the signals. GNSS satellite systems are based on a Circularly Polarized (CP) antennas. Their characteristics include light weight, low profile, low cost, ease of fabrication and ease of integration with circuits which are most favorable parameters for antenna design [2].

\subsection{DESIGN STRATEGIES: DUAL BAND}

For GNSS applications normally dual and tri band antennas are designed. Various design techniques to achieve dual band have been illustrated in [4-7]. The most commonly used design structure is stacked micro strip patch, which provides ease to obtain circular polarization in narrow band but with complex feeding networks or with dual feed configurations $\mathrm{CP}$ can be achieved.

\subsection{TRI-BAND ANTENNA: AN OBVIOUS CHOICE}

GPS antenna must meet the requirements of operation standards vis-a-vis wideband impedance bandwidth with low Axial Ratio (AR) and sufficient Right-Hand Circular Polarization (RHCP) gain with directional radiation pattern toward the upper hemisphere. Therefore, in order to achieve high-precision GPS service, the tri-band (L1, L2, and L5) antenna is essential [1]. The IRNSS receiver is also equipped with tri-band antenna in which GPS L1 band has been incorporated along with IRNSS L5 and S band. This tri-band of IRNSS makes it difficult to design antenna catering. 


\subsection{DESIGN STRATEGIES: TRI-BAND}

Integrating three bands on a single antenna itself is a challenge for portable GPS applications. Again stacked two layer structure is proposed in [8-9] for tri band reception of GPS signals. As per [8], CP is achieved by the multiple slots on ground plane. Top patch resonates at upper band (L1) and bottom patch covers lower two bands (i.e. L2 and L5) which act as parasitic element. However, as cited in [9], three bands are received without any tuning techniques. The antenna is fed by microstrip feeder and high permittivity dielectric materials are used to reduce the antenna dimensions.

A cavity backed tri-band GPS antenna is proposed in [1]. Here, $\mathrm{RHCP}$ is achieved by two meander-line radiators of $1 \lambda \mathrm{g}$ length $(\lambda \mathrm{g}$ : guided wavelength) and an additional meander line radiator of $\lambda \mathrm{g} / 2$ to connect two $1 \lambda \mathrm{g}$-length meander line radiators. Higher gain is achieved by single fed two element meander line arrays as depicted in Fig. 1. This design yields wide impedance bandwidth (VSWR < $2)$, high RHCP gain ( $>4 \mathrm{dBic})$, and wide $\mathrm{AR}(<3 \mathrm{~dB})$ bandwidth within a volume $\left(120 \times 120 \times 29 \mathrm{~mm}^{3}\right)$ [1].

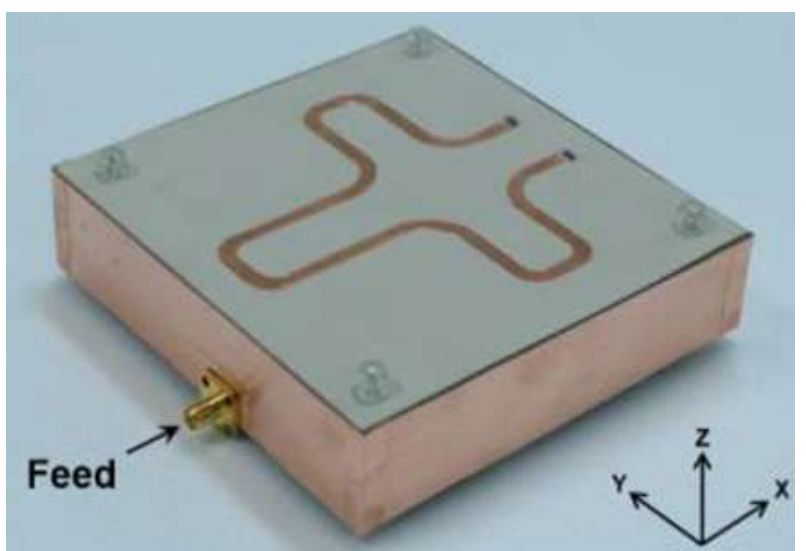

Fig.1. Cavity backed travelling wave antenna: Fabricated view [1]

A planar tri band antenna design is proposed in [10]. Here, low permittivity substrate aims to bring out reduction in quality factor and increase in bandwidth. Tri band is achieved by embedding slot in the main patch to create dual band antenna; doubling the main patch resonances by using two parasitic gap coupled resonators and creating a new band using a single parasitic gap coupled resonator.

Planar monopole antenna is regarded as one among few competent candidates for wireless applications due to the fact that it holds some distinct benefits such as bandwidth, omnidirectional radiation pattern, low profile and low cost. Furthermore, it claims to offer a chance of multi-band operations. There are multiple ways by which multiband reception can be achieved like multi-branched strips are added to the monopole design or slots are cut into radiator or ground plane to excite multiple resonant modes [11-13] but it leads to a large volume or requires a large ground plane.

Recently, Metamaterials have started exhibiting their potentiality in the implementation of resonant antenna and led a new dimension in antenna designing. Using Metamaterial structures in patch antenna, enhanced antenna properties can be yielded as well as size of the antenna can be reduced for convenience. Authors in [14] and [15] have designed monopole tri band antenna using Metamaterial. As cited in [16], two compact dual band antennas have been designed, each having their own tri band reception using Metamaterial for wireless and Global System for Mobile (GSM) applications.

Aforesaid literature survey draws attention towards bottleneck issues and drawbacks that antennas possess like micro-strip antenna has low bandwidth. In order to obtain circular polarization in micro-strip antenna single or double feeding methods can be explored as cited in [2], which in turn increases the size of the antenna that is not preferable. In stark contrast with micro-strip and other aforementioned antenna, spiral antenna is a potential candidate exhibiting certain benefits like it is inherently a circularly polarized antenna.

On the other hand, spiral is frequency independent antenna which can operate at high bandwidth too. Other important aspects of spiral antenna include easy impedance matching, superior radiation efficiency, high spectral efficiency and constant gain which make it a superior choice in contrast with its opponents. Preference of spiral over other antenna can be advocated because number of turns, spacing between them and width of arms can be varied to improve the overall performance. It can be witnessed that preparing an array of spiral ultimately helps in enhancing the gain.

\section{SPIRAL ANTENNA}

Literature review reveals four different classification of spiral antenna namely Archimedean spiral, Square spiral, Star spiral and Log periodic spiral, as illustrated in Fig. 2.

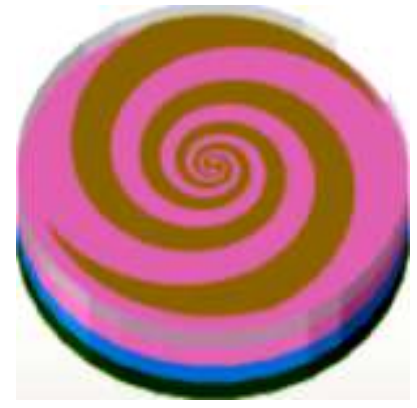

(a) Log periodic Spiral

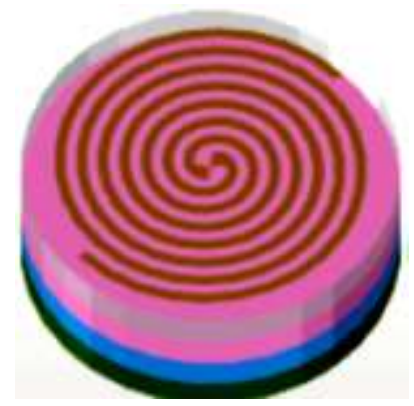

(c) Archimedian Spiral (2 arm)

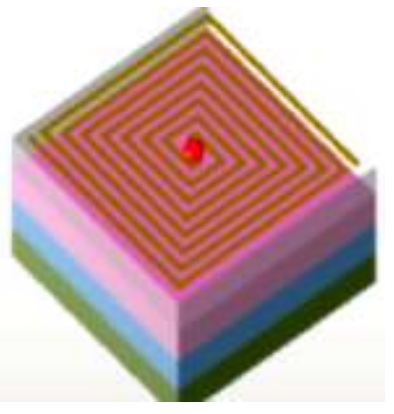

(b) Square Spiral

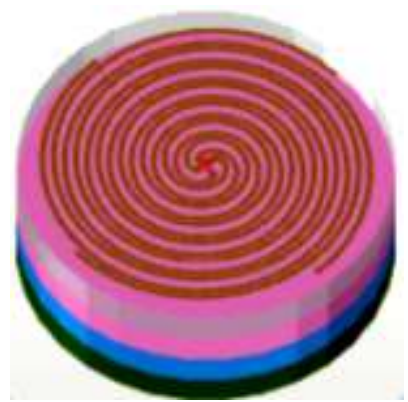

(d) Archimedian Spiral (4 arm)
Fig.2. Types of Spiral Antenna [3]

It operates in 3 ways:

(a) Travelling wave: Formed on spiral arms and allows for broadband performance 
(b) Fast wave: caused due to mutual coupling phenomenon occurring between arms of spiral.

(c) Leaky wave: it leaks the energy during propagation through the spiral arms [3].

\subsection{DESIGN PRINCIPLES OF SPIRAL ANTENNA}

\subsubsection{Equiangular Spiral Antenna:}

Equiangular spiral antenna design is demonstrated in Fig.3. The two arms have an identical geometry, given by:

$$
\begin{gathered}
\rho_{1}=R_{0} e^{a \emptyset} \\
\rho_{2}=R_{0} e^{a(\emptyset-\delta)}
\end{gathered}
$$

where, $\rho_{1}$ and $\rho_{2}$ are respectively the inner and outer curves of antenna's arm, $a$ is the arm growth ratio, $R_{0}$ is the initial inner radius of the arm for $\varnothing=0$ and $\delta$ is the rotation angle between the curves that describes the arm. The other arm of the antenna is described by the same equations rotated by an angle of $\pi$ radians. Good performance of the antenna are achieved when structure is self-complementary. Thus, the rotation angle of the arm has a fixed value $(\delta=\pi / 2)$.

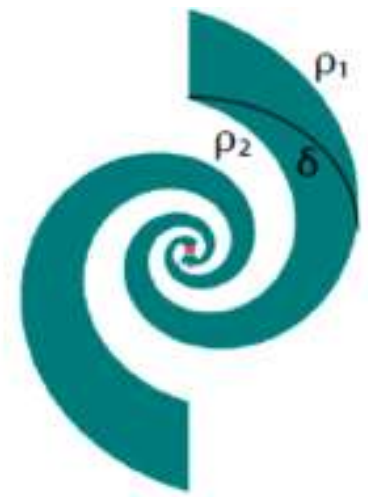

Fig.3. Equiangular spiral antenna [17]

\subsubsection{Lowest Frequency $\left(\lambda_{\text {low }}\right)$ :}

The outer radius of the antenna whose dependence on the wavelength corresponding to the lowest frequency $\left(\lambda_{\text {low }}\right)$ is given by,

$$
R_{\text {out }}=\lambda_{\text {low }} / 2 \pi=c / 2 \pi f_{\text {low }}
$$

where, $c$ is the velocity and is given by

$$
c=c_{o} / \sqrt{ } \varepsilon_{e f f}
$$

$\varepsilon_{e f f}$ is given by,

$$
\varepsilon_{\text {eff }}=\left(\varepsilon_{r}+1\right) / 2
$$

Another important design parameter is the arm length. It is well-known that the range of frequencies where the antenna is circularly polarized begins at the frequency where the total length of the arm is equal to the corresponding wavelength,

$$
L=\lambda_{\text {low }}=c / f_{\text {low }}=\sqrt{ }\left(1+a^{-2}\right)\left(R_{\text {out }}-R_{o}\right)
$$

\subsubsection{Highest Frequency:}

The highest frequency in the spiral antenna's operating band occurs when the innermost radius of the spiral (i.e. where the spiral starts after the feed structure) is equal to $\lambda / 4$ (one quarter wavelength). The highest frequency can be determined from the inner radius,

$$
f_{\text {upper }}=c / \lambda_{\text {upper }}=c / 4 R_{o}
$$

\subsubsection{Input Impedance:}

The input impedance of self-complementary equiangular spiral antenna has a well-known value shown from Babinet's principle as follows,

$$
Z_{\text {ant }}=\eta / 2=120 \pi /\left(2 \varepsilon_{\text {eff }}\right)
$$

\subsubsection{Length of the Structure:}

The length $\mathrm{L}$ of the structure is related to the frequency as,

$$
L=\lambda_{\min } / 4
$$

where, $\lambda_{\min }$ is the wavelength corresponding to the lowest operational frequency.

\subsubsection{Thickness of Substrate:}

Thickness of Dielectric substrate is highlighted by,

$$
h=\lambda / 4
$$

In above equation, $\lambda$ is the wavelength corresponding to the lowest operational frequency of the antenna.

\subsection{DESIGN STRATEGIES OF SPIRAL AS DUAL/TRI BAND ANTENNA}

As cited in [17], the equiangular spiral antenna for extremely high frequencies is proposed which performs at $65 \mathrm{GHz}$ and $170 \mathrm{GHz}$. Simulation is carried out using the Ansoft Designer. It is advocated that better directivity is obtained for antennas with smaller arm growth rate by observing different values of arm growth rate. To provide balanced feeding to antenna, the tapered balun was designed which transforms an unbalanced feeding point such as a micro-strip line to a balanced feeding point such as parallel slot lines at the same time it also provides impedance matching between the source and the spiral antenna. Such design is depicted in Fig.4.

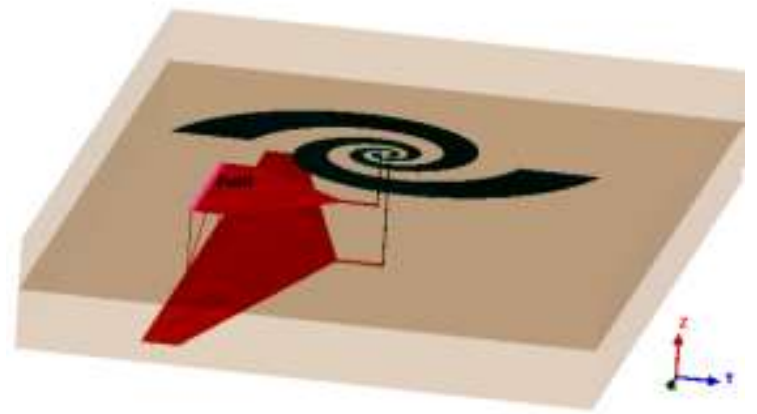

Fig.4. Equiangular Spiral Antenna with Linear Tapered Balun [17]

The dual band dual polarized antenna is proposed as shown in Fig.5 for the Chinese Compass Navigation Satellite System (CNSS) [18]. This antenna performs at L band $(1616+/-5 \mathrm{MHz})$ which is left-handed circularly polarized and at $S$ band $(2492+/-$ $5 \mathrm{MHz}$ ) and is right-handed circularly polarized. In this paper the single arm spiral with large slot and a stepped micro-strip feedline is designed as the inner embedded spiral slot can improve the axial ratio bandwidth and by adjusting the width and length of the micro-strip feed-line the matching for the two bands is improved.

The coaxial-fed Rectangular Spiral Micro-strip Antenna (RSMA) for Wi-Fi application is proposed with bandwidth $22 \mathrm{MHz}$ and the center frequency is $2.45 \mathrm{GHz}$ [19]. A commercial $3-D$ electromagnetic simulator is used to simulate the design. The antenna fabricated on FR4 substrates with 4.9 dielectric constant 
and $1.54 \mathrm{~mm}$ thickness and the size is $6 \mathrm{~cm} \times 6 \mathrm{~cm}$. The bottom coaxial fed provide space for other device to mount on the substrate which can further reduce the overall size of a Wifi.

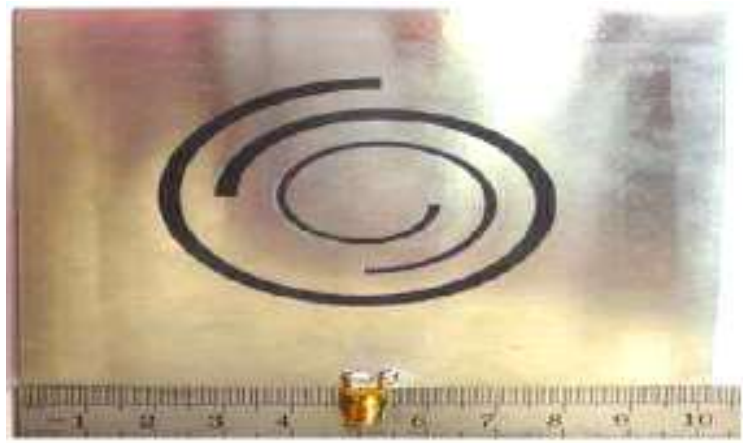

Fig.5. Spiral Antenna Design for CNSS [18]

The work reported in [20] describes a two-arm unbalancedexcitation spiral antenna, where one arm is fed by the inner conductor of a coaxial line and the other is short-circuited to a conducting cavity acting as a parasitic element that backs the spiral. The range of operating frequency is $1 \mathrm{GHz}$ to $10 \mathrm{GHz}$. This is used as wideband CP antenna. Input impedance and gain of this antenna is smaller than that for the corresponding balanced excitation spiral. The radiation pattern is along $\mathrm{Z}$-axis and circularly polarized.

As stated in [21], author describes the axial ratio characteristics of an Archimedean spiral antenna placed on a thin magnetic material. Antenna under test has improved axial ratio (AR) and shorted arm length. Archimedean spiral antenna explored in this work is a potential candidate in designing ultra-wideband systems because of its superior performance on circular polarization while maintaining consistent gain and input impedance. Also, it is a popular choice among various frequency independent antennas. The active region of spiral is the region from where the circumference of the spiral equals one wavelength. The fabricated view of such antenna is depicted in Fig.6.

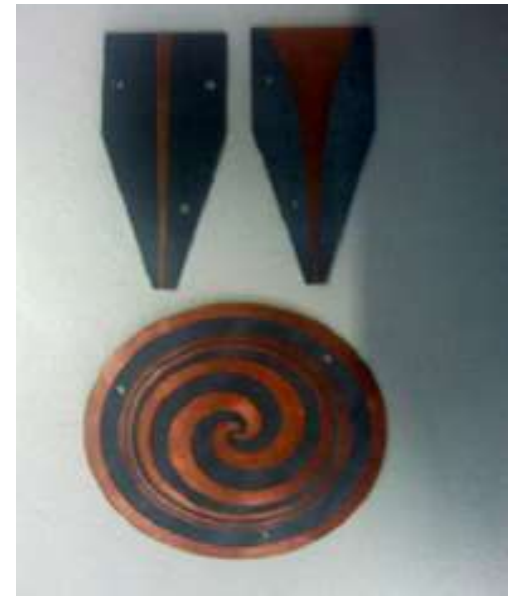

(a)

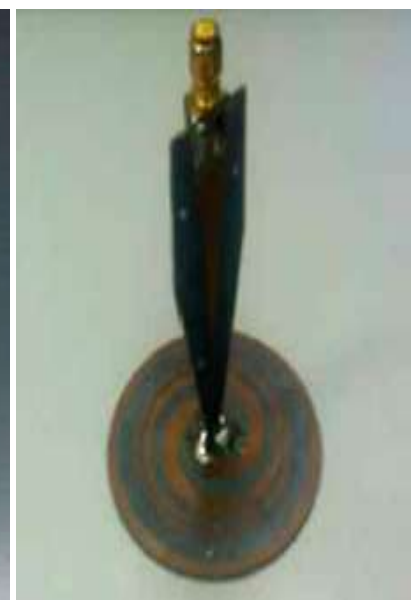

(b)
Fig.6. Archimedean Spiral Antenna (a) Antenna and Balun (b) Fabricated View [21]

Further, Three-Band Multi-polarization Cross Spiral electrically small antenna is reported in [22] which radiates CP of a GPS band simultaneously with Linear Polarization (LP) of
Radio Frequency IDentification (RFID) band and a mobile phone band. Multi-polarization is accomplished by using a Dipole-Fed Cross Spiral Antenna (DF-CSA) and electrically small structure is attained by adding fork-shape elements to the DF-CSA. Suggested design is highlighted in Fig.7 and is 35 square millimeters and radiates omni-directional $\mathrm{CP}$ with $0 \mathrm{dBi}$ or more gain and $62 \%$ or more efficiency within $28 \mathrm{MHz}$ band-width.

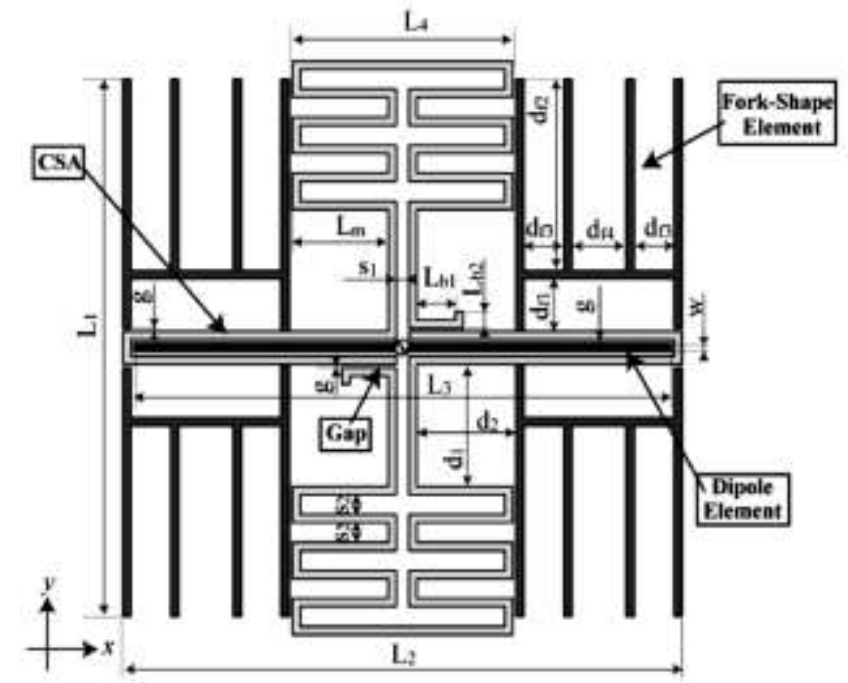

Fig.7. Top view of Cross Spiral Antenna [22]

As proposed in [23], antenna using Artificial Bee Colony (ABC) algorithm for passive RFID tag application at Ultra High Frequency (UHF) band. For aforesaid work, optimization goals are antenna size minimization, gain maximization, conjugate matching also in consequence the maximization of the read range. Antenna dimensions were optimized and evaluated with commercial Electro-Magnetic (EM) software. Complete design structure is illustrated in Fig.8. It can be witnessed that the developed $\mathrm{ABC}$ algorithm works more efficiently in contrast with their counterparts. The design of passive RFID tag antennas is found efficient due to following reasons: antennas with dimensions less than $3 \mathrm{~cm}$, gain reaches the value of $1.6 \mathrm{dBi}$ and read range about $6 \mathrm{~m}$. Above, simulation is carried out on MTI Wireless Edge/MT-242032/NRH.

Spiral antenna at a frequency range between 2.4 to $2.5 \mathrm{GHz}$ is designed and developed that is fed by a micro-strip line and RT Duroid 5870 as reported in [24]. Such antenna is designed with dielectric constant $\varepsilon_{r}$ and thickness $t_{s u b}$ of 2.33 and $0.5 \mathrm{~mm}$ respectively. Simulation and analysis of above antenna is carried out with Genesys. A prototype of a circular microstrip antenna is fabricated and measured that consists of one arm which forms the spiral structure. The arm has width $w$ and the separation between the lines is described by the parameter $s$. The number of turns of the middle line is denoted by $N$ and the maximum radius is specified by $r_{\text {out }}$. Input impedance of the antenna is affected by the line width $w$, the distance between the lines $s$, the dielectric constant $\varepsilon_{r}$ and the substrate thickness $t_{s u b}$. The radiation efficiency of the patch antenna depends largely on the permittivity $\left(\varepsilon_{\mathrm{r}}\right)$ of the dielectric. Followings are the four most popular feed techniques: micro-strip line, coaxial probe (both contacting schemes), aperture coupling and proximity coupling (both noncontacting schemes). This spiral design makes use of micro-strip line feed technique. For ideal antenna, value for VSWR is equal 
to 1 having no reflection. Low values of VSWR indicate the antenna have a small value of reflection coefficient. Snapshot of the fabricated antenna is showcased in Fig.9.

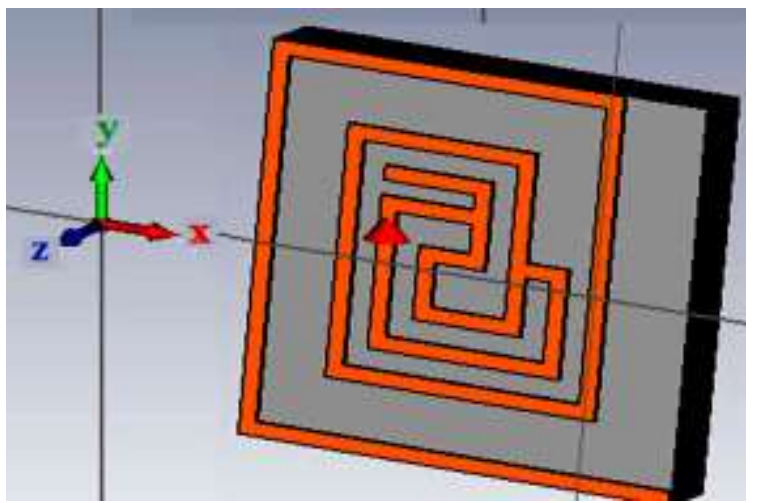

Fig.8. Spiral Antenna Structure for RFID tag geometry [23]

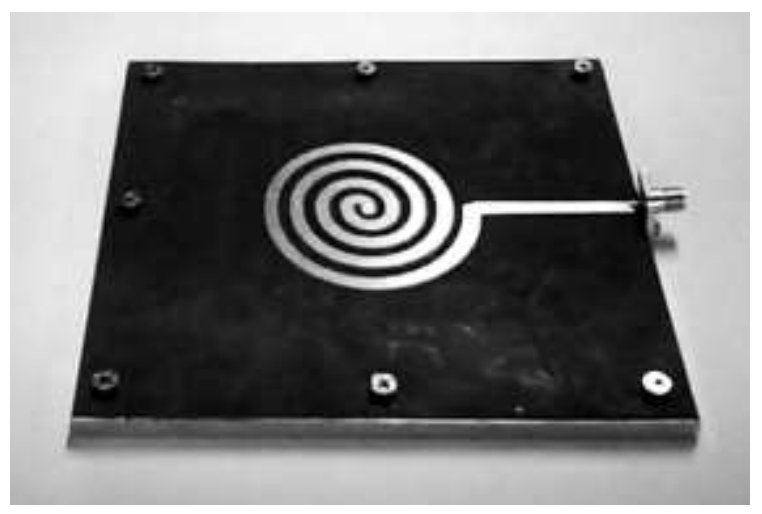

Fig.9. A single arm spiral antenna with Micro-strip Line Feed [24]

\section{CONCLUSION}

Wireless systems involved in transmission and reception of electromagnetic waves envisage that efficient and effective designing of antenna is considered to be potential material of research for quite some time amongst scientific community and off late added a new dimension of research and development.

Prerequisites for developing antenna for GNSS applications (especially IRNSS) include circular polarization, frequency independence and reconfigurable nature. In order to cater the above needs, out of all potential candidates, spiral antenna is proven to be most promising while fulfilling all requirements.

Looking to the future demands for navigation, mapping, accurate positioning and other applications, IRNSS is expected to play a vital role. This paper touches upon various designing aspects of spiral antenna where inherent freedom of varying number of turns, spacing between them and width of arm is quite evident. The gain of spiral antenna can be increased considerably by forming an array. Even the performance of such antenna can be improved by improving axial ratio and reducing arm length. Thus, review carried out in this paper investigates and further advocates supremacy of spiral antenna in comparison with others and eventually provides a step forward for the researchers to contribute in designing dual / tri band spiral based antenna for various GNSS applications including IRNSS.

\section{REFERENCES}

[1] Yonghyun Yoon and Byungje Lee. "A Cavity-backed Traveling Wave Antenna for Tri-Band GPS Applications", IEEE Antennas and Wireless Propagation Letters, Vol. 15, pp. 1454-1457, 2016.

[2] M. Karthick and K.R. Kashwan, "Design of IRNSS Receiver Antenna for Smart City Applications in India", Proceedings of IEEE Global Conference on Communication Technologies, pp. 277-280, 2015.

[3] S. Patil, C. Vyas and A. Khalore. "Spiral Antennas for Communication Engineering Applications: A Systematic Approach", International Journal of Innovative Research in Electronics and Communication, Vol. 3, No. 2, pp. 1-6, 2016.

[4] David M. Pozar and Sean M. Duffy, "A Dual-Band Circularly Polarized Aperture-Coupled Stacked Microstrip Antenna for Global Positioning Satellite", IEEE Transactions on Antennas and Propagation, Vol. 45, No. 11, pp. 1618-1625, 1997.

[5] Kush Agarwal, Yong Xin Guo and Arokiaswami Alphones, "Dual-Band Circularly Polarized Stacked Microstrip Antenna over RIS for GPS Applications", Proceedings of IEEE Wireless Symposium, pp. 1-4. 2013.

[6] Stephane Bila Chantalat and Bernard Jarry, "Low-Profile Dual-Band Circularly Polarized Microstrip Antenna for GNSS Applications", Proceedings of $9^{\text {th }}$ IEEE European Conference on Antennas and Propagation, pp. 1-4, 2015.

[7] Xiaoye Sun, Zhijun Zhang and Zhenghe Feng, "Dual-Band Circularly Polarized Stacked Annular-Ring Patch Antenna for GPS Application", IEEE Antennas and Wireless Propagation Letters, Vol. 10, pp. 49-52, 2011.

[8] Yoonjae Lee, Suman Ganguly and Raj Mittra, "Tri-Band (L1, L2, L5) GPS Antenna with Reduced Backlobes", Proceedings of $28^{\text {th }}$ General Assembly of International Union of Radio Science, pp. 1-5, 2005.

[9] Maher M. Abd Elrazzak and M.F. Alsharekh, "A Compact Wideband Stacked Antenna for the Tri-Band GPS Applications", Active and Passive Electronic Components, Vol. 2008, pp. 1-4, 2008.

[10] Michal Pokorny, Jiri Horak and Zbynek Raida, "Planar TriBand Antenna Design", Available at: https://dspace.vutbr.cz/bitstream/handle/11012/57175/08_0 1_28_36.pdf?sequence=1.

[11] J. Chen, S.T. Fan, W. Hu and C.H. Liang, "Design of Triband Printed Monopole Antenna for WLAN and WIMAX Applications", Progress in Electromagnetics Research C, Vol. 23, pp. 265-275, 2011.

[12] Le-Hu Wen, Ying-Zeng Yin, Zhen-Yang Liu, Dan Xi, Min Zhang and Yan Wang, "Performance Enhancement of TriBand Monopole Antenna for WLAN/WiMAX Applications", Progress in Electromagnetics Research Letters, Vol. 15, pp. 61-68, 2010.

[13] Gehan Shehata, Mahmoud Mohanna and Mohammed Lotfy Rabeh, "Tri-Band Small Monopole Antenna based on SRR Units", NRIAG Journal of Astronomy and Geophysics, Vol. 4, No. 2, pp. 185-191, 2015.

[14] Guo-Hong Du, Xiaohong Tang and Fei Xiao, "Tri-Band Metamaterial-Inspired Monopole Antenna with Modified S- 
Shaped Resonator", Progress in Electromagnetics Research Letters, Vol. 23, pp. 39-48, 2011.

[15] Jiang Zhu, Marco A. Antoniades and George V. Eleftheriades, "A Compact Tri-Band Monopole Antenna with Single-Cell Metamaterial Loading", IEEE Transactions on Antennas and Propagation, Vol. 58, No. 4, pp. 1031-1038, 2010.

[16] Maryam Rahimi, Ferdows B. Zarrabi, Rahele Ahmadian, Zahra Mansouri and Asghar Keshtkar, "Miniaturization of Antenna for Wireless Application with Difference Metamaterial Structures", Progress in Electromagnetics Research, Vol. 145, pp. 19-29, 2014.

[17] Bogdan Sirbu, Tolga Tekin and David Pouhe, "Design and Simulation of an Equiangular Spiral Antenna for Extremely High-Frequencies", Proceedings of $8^{\text {th }}$ IEEE European Conference on in Antennas and Propagation, pp. 31023106, 2014.

[18] Hangying Yuan, Shaobo Qu, Jieqiu Zhang, Hang Zhou, Jiafu Wang, Hua Ma, and Zhuo Xu, "Dual-Band DualPolarized Spiral Antenna for Chinese Compass Navigation Satellite System", Progress in Electromagnetics Research, Letters, Vol. 46, pp. 25-30, 2014.

[19] N.H. Abdul Hadi, K. Ismail, S. Sulaiman and M.A. Haron, "Design of a Rectangular Spiral Antenna for Wi-Fi
Application", Proceedings of $13^{\text {th }}$ International Conference on Advanced Communication Technology, pp. 30-34, 2011.

[20] Magnus Karlsson and Shaofang Gong, "An Integrated Spiral Antenna System for UWB”, Proceedings of European Microwave Conference, pp. 1-4, 2005.

[21] Hui Fen Huang and Zonglin Lv, "A New Spiral Antenna with Improved Axial Ratio and Shorted Arm Length", Progress in Electromagnetics Research C, Vol. 46, pp. 8389, 2014.

[22] Mayumi Matsunaga and Masataka Suzuki, "An Electrically Small Three-Band Multi-Polarization cross Spiral Antenna", Proceedings of $9^{\text {th }}$ European Conference on In Antennas and Propagation, pp. 1-2, 2015.

[23] Sotirios K. Goudos, Katherine Siakavara, and John N. Sahalos, "Novel Spiral Antenna Design using Artificial Bee Colony Optimization for UHF RFID Applications", IEEE Antennas and Wireless Propagation Letters, Vol. 13, pp. 528-531, 2014.

[24] M.F. Abdul Khalid, M.A. Haron, A. Baharudin and A.A. Sulaiman, "Design of a Spiral Antenna for Wi-Fi Applications", Proceedings of IEEE International Conference on RF and Microwave, pp. 431-435, 2008. 